\title{
The Role of Palliative Care in Oncology
}

\author{
Rajiv Agarwal, $\mathrm{MD}^{1} \quad$ Andrew S. Epstein, $\mathrm{MD}^{2,3}$ \\ ${ }^{1}$ Department of Medicine, Medical Oncology, Memorial Sloan \\ Kettering Cancer Center, New York, New York \\ 2 Division of Solid Tumor Oncology, Department of Medicine, \\ Gastrointestinal Oncology Service, Memorial Sloan Kettering \\ Cancer Center, New York, New York \\ ${ }^{3}$ Department of Medicine, Palliative Medicine Service, Memorial \\ Sloan Kettering Cancer Center, New York, New York \\ Semin Intervent Radiol 2017;34:307-312
}

\begin{abstract}
Address for correspondence Andrew S. Epstein, MD, Gastrointestinal Oncology Service, Palliative Medicine Service, Memorial Sloan Kettering Cancer Center, 300 E. 66th Street, Room 1013, New York, NY 10065 (e-mail: epsteina@mskcc.org).
\end{abstract}

\begin{abstract}
Keywords

- palliative care

- oncology

- interventional radiology

Palliative care is a powerful adjunct to oncology that adds distinct value to the physical, mental, and psychosocial well-being of patients living with cancer. Its expanding role and integration with standard oncologic care has proven clinical benefit, as the practice of palliative care can help alleviate symptom burden, enhance illness and prognostic understanding, and improve both the quality of life and overall survival for patients. The primary aim of this review article is to highlight the significant interplay between palliative care and oncology and, in doing so, shed light on the areas for improvement and modern challenges that exist to meet the complex palliative care needs of patients with cancer.
\end{abstract}

Objectives: Upon completion of this article, the reader will be able to discuss the role of interventional radiology in the palliative treatment of terminally ill patients, including the societal need for such care, current obstacles to such care, and the need for close integration of care with referring care provider.

Accreditation: This activity has been planned and implemented in accordance with the Essential Areas and Policies of the Accreditation Council for Continuing Medical Education (ACCME) through the joint providership of Tufts University School of Medicine (TUSM) and Thieme Medical Publishers, New York. TUSM is accredited by the ACCME to provide continuing medical education for physicians.

Credit: Tufts University School of Medicine designates this journal-based CME activity for a maximum of $\mathbf{1} \boldsymbol{A M A}$ PRA Category 1 Credit $^{\mathrm{TM}}$. Physicians should claim only the credit commensurate with the extent of their participation in the activity.

Palliative care is an integral component of the comprehensive care for patients in oncology. Its clinical practice is rooted in the philosophy of improving the quality of life for patients who are living with serious illnesses and for their families. In doing so, this interdisciplinary specialty aims to alleviate symptom burden, treat spiritual and psychological distress, enhance illness and prognostic understanding to establish goals of care, and help patients cope with their condition and the dying process as they transition to the end of life. ${ }^{1,2}$ Though palliative care physicians traditionally provide hospice and end-of-life care for patients during the last months of life, the expanding role and core concepts of palliative medicine are vital from the time of diagnosis-even when cure is possibleand should be integrated throughout the trajectory of a serious medical condition, such as cancer.

This review article is not intended to be a comprehensive assessment of the role of palliative care for cancer patients, but rather aims to highlight the importance and related challenges of integrating palliative care in our modern era of oncology. Herein, we plan to address the following salient topics: (1) a brief overview of measuring symptom burden; (2) the often unrealistic expectations and inaccurate understanding of illness of patients with advanced disease; (3) the evidence which support models of integration and highquality primary palliative care; (4) the barriers to meeting a growing demand for palliative care; (5) palliative care in the dynamic landscape of cancer therapeutics; and (6) the
Issue Theme Palliative Care Interventions in the Cancer Patient; Guest Editors, David Li, MD, PhD and David C. Madoff, MD
Copyright @ 2017 by Thieme Medical Publishers, Inc., 333 Seventh Avenue, New York, NY 10001, USA. Tel: +1(212) 584-4662.
DOI https://doi.org/ 10.1055/s-0037-1608702. ISSN 0739-9529. 
expanded role of interventional radiology (IR) as a specialty in procedural palliative care. The primary objective of this review is to improve physician awareness of the unmet palliative care needs for patients with cancer and, ultimately, elucidate the synergistic effect that palliative medicine can have along with cancer-directed treatment on the physical and mental well-being of patients.

\section{Symptom Burden and Patient-Reported Outcomes}

According to the National Consensus Project for Quality Palliative Care, the domains of palliative care and recommendations for optimal delivery include the evaluation, support, and treatment of the physical, psychological, social, spiritual, and cultural aspects of care, while also assisting patients in the coordination of care and shared decision-making process throughout their disease trajectories., ${ }^{3,4}$ Guidelines for incorporating these principles of palliative care into oncology practice help inform oncologists how to longitudinally assess patients' values and treatment goals, and concurrently intervene to manage their cancer-related symptoms.

Some of the common physical manifestations of cancer include pain, anorexia, cachexia, fatigue, nausea, constipation, dyspnea, and malignant bowel obstructions. These physical symptoms as well as the related spiritual and psychosocial distress during a patient's treatment course are routinely addressed by both medical oncologists and palliative care specialists in clinic visits. Recent clinical studies that evaluate symptom monitoring with web-based patient-reported outcomes have shown an added clinical benefit, empowering patients to take ownership of their disease and allowing for earlier detection of symptom recurrence.

Basch et al demonstrated that patients initiating chemotherapy who are randomized with an ability to notify their health care teams with interval symptoms have a higher quality of life and improved overall survival compared with patients who receive usual care. ${ }^{5}$ Differences in survival may be attributed to early detection and, consequently, prompt management of disease and treatment-related toxicities. A similar study in patients with high-risk lung cancers showed that an electronic-based follow-up evaluation for symptom burden can improve clinical outcomes, with identifying the need for earlier supportive care and thereby improving quality of life and survival. ${ }^{6}$ These studies collectively indicate how oncologists and palliative care specialists can monitor the symptoms of patients with cancer, both in clinic and by patient-driven reports.

\section{Expectations and Illness Understanding}

Along with helping to relieve symptoms, palliative care in oncology is deeply involved in supporting patients as they cope with and comprehend their disease, and the goals of treatment. Chemotherapy is offered in the metastatic setting with the goal of improving symptoms and stabilizing disease. Poor understanding of the goals of treatment for incurable metastatic disease can compromise the ability of patients to make informed treatment decisions, and ultimately may delay end-of-life care and planning. Prior studies have shown that patients' decisions to receive treatment for advanced stage illnesses are dependent on their knowledge of the likelihood of adverse outcomes and overall burden of the treatment itself-including length of hospital stays, frequency and degree of invasive interventions, and extent of monitoring. ${ }^{7}$ Yet, a pivotal secondary analysis of data from the Cancer Care Outcomes Research and Surveillance (CanCORS) study showed that $69 \%$ of patients with stage IV lung cancer and $81 \%$ of patients with stage IV colorectal cancer who elected to receive systemic treatment had inaccurate expectations for the curative potential of chemotherapy. ${ }^{8-10}$ Supplementary findings have shown that patients with advanced cancers who did not expect chemotherapy to cure their disease still received treatment at similar rates to those patients who had higher expectations, but were more likely to enroll in hospice services before death. ${ }^{11}$

Patient clinical encounters, and both the content and manner in which prognostic information is disclosed, have significant impact on how patients make treatment-related decisions. Discordance between patients and oncologists with regard to prognosis and expectations for treatment remain common. ${ }^{12}$ Epstein et al have shown that more accurate illness understanding is associated with patient reports that discussions have occurred on prognosis and life expectancy with their oncologists. ${ }^{13}$ In this study, illness understanding scores were generated for patients with advanced cancers refractory to standard chemotherapy who were expected to die within 6 months, based on four variables: patient acknowledgment of terminal illness, recognition of incurability, knowledge of advanced stage of disease, and accurate expectations to live months as opposed to years. In comparison to patients who never participated in or only had remote conversations about prognosis with their oncologists, patients involved in more recent conversations had higher illness understanding scores and better insight into the terminal nature of their metastatic cancers. In addition, communicating information with varying degrees of optimism correlates with patients' perceptions of physician compassion, as content presented in a more optimistic manner is often associated with higher levels of perceived physician compassion and trust. ${ }^{14}$ Communication skills training on discussing life expectancy may be of benefit for oncologists to facilitate accurate patient comprehension of the goals of treatment for incurable disease, all the while maintaining a therapeutic relationship and a message of hope. ${ }^{15}$

Along with a dedicated focus by oncologists to clarify any illness misconceptions patients may have, specialty palliative care can serve as an adjunct to help patients mentally prepare for the role and appropriate utilization of systemic cancer treatment. In patients with advanced non-small cell lung cancer, those randomized to receive early concurrent palliative care with oncologic care compared with those who received standard oncologic care alone developed a more accurate understanding of their prognosis over time, and were less likely to receive systemic chemotherapy at the end 
of life. ${ }^{16}$ Of note, baseline perceptions on the curability of terminal cancers and goals of therapy prior to randomization did not differ between study groups, and confirmed that the majority of patients with metastatic disease continue to expect curative outcomes. Oncologists and palliative care physicians therefore must continue to work in tandem, with dual clarity and compassion, toward sustained illness understanding to help patients make informed decisions, accept the realistic goals of treatment, and plan for end-of-life care.

\section{Models of Integration of Palliative Care}

Traditional palliative care integration by in-hospital consultations and end-of-life conversations has been shown to improve the quality of death for patients with advanced cancer and, in doing so, reduce health care costs and utilization. ${ }^{17,18}$ It is undeniable that systemic chemotherapy without clear benefit for patients at the end of life results in adverse toxicities, delays hospice referrals, and does not improve survival. ${ }^{19-21}$ Of equal importance, it adds to illness misunderstanding and prevents patients from mentally and emotionally preparing for the dying process. Given the proven value of palliative care in oncology for symptom management, quality of life, and prognostic understanding of terminal illnesses, there has been growing evidence in the last decade to support the early integration of palliative care for cancer patients.

Recent clinical trials examining the effects of early integration of specialty palliative care show marked improvement in patient satisfaction, mood, quality of life, health care utilization, and overall survival. For example, patients who received early in-home palliative care services in addition to usual care had fewer emergency department visits, hospital admissions, and lower medical costs. ${ }^{22}$ The ENABLE II randomized controlled trial showed that patients with terminal cancer who received early supplementary palliative care administered by advanced practice nurses had better quality of life and mood. ${ }^{23}$ Research by Zimmerman et al on the early involvement of ambulatory palliative care for patients with metastatic cancer further demonstrated superior outcomes in patient satisfaction. ${ }^{24}$ In a landmark randomized controlled trial by Temel et al, patients with metastatic nonsmall cell lung cancer were assigned to receive either standard oncologic care alone or standard oncologic care with early concurrent palliative care near the time of diagnosis. ${ }^{25}$ This study not only validated prior research that the addition of palliative care improved quality of life and reduced depression, but notably it reported a statistically significant difference in overall survival between study groups. The median survival of patients randomized to early palliative care was 11.6 months, whereas the median survival of patients receiving standard oncologic care alone was 8.9 months $(p=0.02$ ). Patients assigned to early palliative care also received less aggressive cancer-directed care, including chemotherapy, at the end of life. ${ }^{25}$ Subsequent studies interestingly show that the effectiveness of early palliative care may vary per cancer type, especially given the differences in baseline disease trajectories and available therapeutic options. ${ }^{26}$
The integration of specialty palliative care therefore has meaningful benefit for patients with advanced cancers and is strongly advocated by the American Society of Clinical Oncology. ${ }^{27}$ However, the practicality of requiring palliative care specialists to address all aspects of palliative medicine is not feasible in our modern era of cancer care, where needs are complex and many. For this reason, primary oncologists should share in the responsibility to provide basic psychosocial support, manage cancer-related symptoms, and help patients with advance care planning and discuss end-of-life preferences. We must establish a model that maximizes the utilization efficiency of health care resources and defines the necessary skill sets to optimally deliver primary and specialty palliative care. ${ }^{28-30}$ Education in core palliative care principles for all levels of learners and providers in oncology will enhance high-quality patient care, and allow for better triage of the complex and refractory palliative care needs to palliative care specialists. Oncologists who seek dual training in palliative care can serve as ambassadors who know how to effectively bridge and understand the nuances of both disciplines. $^{31}$

\section{Meeting the Demand}

One of the pitfalls for relying on specialty palliative care in oncology is that the current workforce of palliative care specialists is insufficient to meet the growing demands of an aging cancer population. The Institute of America's influential 2014 publication on Dying in America: Improving Quality and Honoring Individual Preferences Near the End of Life outlines the need and ensuing challenges for focused improvement in physician training in palliative care in our current health care system. ${ }^{32}$ Prior surveys conducted by Hui et al in 2010 have demonstrated that most NCI-designated and non-NCI cancer centers have palliative care services, but vary in the extent of their utilization and success of their integration. ${ }^{33}$ To provide cancer patients with high-quality palliative care, more recent studies seek to characterize the current state and scope of palliative care services offered at national cancer centers. A 2015 NCCN survey indicated that all responding institutions have inpatient consultation services and majority have outpatient clinics to address symptom management and end-of-life care; however, only $23 \%$ have homebased palliative care services and that $80 \%$ of these institutions still report inadequate capacity to meet demand. ${ }^{34}$ While this survey illustrates the increasing prevalence of outpatient palliative care among cancer centers, there remain opportunities for much needed improvement. The supply-demand gap in palliative care is largely due to a lack of trained specialists, and further investment in training all members of a patient's oncology team with primary palliative care skills has the potential to lessen this imbalance. $^{35}$ Moreover, while inpatient consultation services have been uniformly available for patients compared with outpatient or home palliative care, few hospitals have appropriate and sufficient staff to comply with national recommendations. ${ }^{36}$ Additional funding and education to 
develop a sustainable model that seamlessly dovetails palliative services with oncologic care will undoubtedly have meaningful clinical and psychological impact for patients and their families.

\section{The Evolving Era of Cancer Therapeutics}

Over the past decade, the rapid and ongoing evolution of cancer therapeutics has changed the landscape of oncology. The advent of immunotherapy to block the inhibitory interactions between cancer cells and host immunity, as well as the development of precision oncology to target individual driver mutations, offers new treatment options that have the potential to extend overall and disease-free survival. However, as investigational therapeutics expand and clinical trial participation increases, oncologists and their patients must contend with the uncertainty of prognostic understanding. ${ }^{37,38}$ This creates an unparalleled challenge for patients to engage in informed conversations with their oncology and palliative care teams, especially with regard to preparing for their future and discussing end-of-life care options. Oncologists and palliative care specialists must directly address these uncertainties with each clinical encounter, with the goal of tempering unrealistic expectations and helping patients navigate their disease trajectories in real time.

Furthermore, patients with advanced disease that progress on standard-of-care treatment options and who elect to participate in phase I investigational trials represent a growing patient population with unmet palliative care needs. ${ }^{39-41}$ Studies which predate the emergence of immunotherapy and molecularly targeted therapy using tumor genomics demonstrate that patients who participate in phase I clinical trials often expect success and underestimate the potential toxicity of experimental therapy compared with standard therapy. ${ }^{42}$ These patients also have increased symptom burden and are less likely to seek home services. ${ }^{43}$ Additional research is needed to evaluate the role of palliative care in patients receiving experimental therapeutics. While enrollment in phase I trials and receipt of palliative care are not mutually exclusive, the promise of translational medicine requires a more concerted effort by physicians to help patients balance hope with accurate illness understanding. ${ }^{44-46}$

\section{Interventional Radiology and Palliative Care}

The discipline of IR has a significant role in the longitudinal care of patients with cancer, from obtaining tissue diagnosis with image-guided biopsies to providing therapeutic interventions and palliation of cancer-related symptoms. The evolution of procedural techniques has enabled interventional radiologists to expand their role in the treatment of cancer, and provide novel ways to alleviate symptom burden, such as refractory pain, beyond conventional therapy. ${ }^{47-49}$ Patients with cancer therefore derive significant benefit from the contribution of IR to the multimodality treatment plan outlined by their primary oncologist.
In particular, the minimally invasive palliative procedures conducted by IR greatly improve the quality of life and relieve suffering for cancer patients. Percutaneous ablative and nerve-block procedures for pain control, vertebroplasty for compression fractures due to osseous lesions, and image-guided interventions to decompress malignant obstructions and drain persistent effusions or ascites are a few examples that demonstrate the powerful impact of IR on the management of cancer-related symptoms. ${ }^{50}$ Given the overwhelming benefit of such interventions, it is important to integrate IR into the multidisciplinary approach to optimizing supportive care in the cancer patient population. ${ }^{51-53}$ Rational decision making for timely palliative interventional procedures during the course of a patient's disease necessitates an open dialogue among all members of a patient's health care team. Moreover, validated disease-specific quality-of-life assessments in the periprocedural setting are helpful tools to select appropriate interventions, and can be used to measure the efficacy of IR procedures on improving patient-reported outcomes and controlling symptoms. ${ }^{54}$

Similar to the paradigm shift of incorporating primary palliative care within medical oncology, there is a greater recognition for the role of primary clinical palliative care for interventional radiologists. Given the dynamic interplay of palliative care and IR, the American Board of Radiology supports adjunctive training and certification in Hospice and Palliative Medicine. Regardless of whether trainees decide to pursue additional training in palliative care formally or informally, interventional radiologists are uniquely positioned to help patients cope with serious illnesses with the use of image-guided interventions. ${ }^{55-57}$ The responsibility to assist in symptom management and recognize how such interventions can enhance the quality of life in patients with advanced malignancies thereby underscores the value of interventional radiologists as procedural palliative care specialists.

\section{Conclusion}

Palliative care is essential for the physical, mental, and psychosocial health of patients with advanced cancer. Its synergistic effect on overall survival, while improving outcomes in patient satisfaction and quality of life, merits its inclusion with standard oncologic care. Ongoing dedicated research is needed to evaluate the integration and expansion of high-quality palliative care services to meet the demands of a growing cancer population. Medical oncology and other specialties, including interventional radiology, must incorporate primary palliative care skills into their practice, and work together with specialty palliative care physicians to help patients better understand and cope with their illnesses-a task that includes, but is not limited to, when such illnesses are terminal.

\section{Acknowledgment}

This research was funded in part through the $\mathrm{NIH} / \mathrm{NCI}$ Cancer Center Support Grant P30 CA008748. 


\section{References}

1 Kelley AS, Morrison RS. Palliative care for the seriously ill. N Engl J Med 2015;373(08):747-755

2 Levy M, Smith T, Alvarez-Perez A, et al. Palliative care version 1.2016. J Natl Compr Canc Netw 2016;14(01):82-113

3 American Academy of Hospice and Palliative Medicine; Center to Advance Palliative Care; Hospice and Palliative Nurses Association; Last Acts Partnership; National Hospice and Palliative Care Organization. National Consensus Project for Quality Palliative Care: Clinical Practice Guidelines for quality palliative care, executive summary. J Palliat Med 2004;7(05):611-627

4 Ferrell B, Connor SR, Cordes A, et al; National Consensus Project for Quality Palliative Care Task Force Members. The national agenda for quality palliative care: the National Consensus Project and the National Quality Forum. J Pain Symptom Manage 2007;33(06):737-744

5 Basch E, Deal AM, Kris MG, et al. Symptom monitoring with patient-reported outcomes during routine cancer treatment: a randomized controlled trial. J Clin Oncol 2016;34(06):557-565

6 Denis F, Lethrosne C, Pourel N, et al. Overall survival in patients with lung cancer using a web-application-guided follow-up compared to standard modalities: results of a phase III randomized trial. Abstract presented at the ASCO 2016 Annual Meeting; June 3-7, 2016: Chicago, IL

7 Fried TR, Bradley EH, Towle VR, Allore H. Understanding the treatment preferences of seriously ill patients. N Engl J Med 2002;346(14):1061-1066

8 Ayanian JZ, Chrischilles EA, Fletcher RH, et al. Understanding cancer treatment and outcomes: the Cancer Care Outcomes Research and Surveillance Consortium. J Clin Oncol 2004;22(15):2992-2996

9 Malin JL, Ko C, Ayanian JZ, et al. Understanding cancer patients' experience and outcomes: development and pilot study of the Cancer Care Outcomes Research and Surveillance patient survey. Support Care Cancer 2006;14(08):837-848

10 Weeks JC, Catalano PJ, Cronin A, et al. Patients' expectations about effects of chemotherapy for advanced cancer. N Engl J Med 2012; 367(17):1616-1625

11 Mack JW, Walling A, Dy S, et al. Patient beliefs that chemotherapy may be curative and care received at the end of life among patients with metastatic lung and colorectal cancer. Cancer 2015;121(11):1891-1897

12 Gramling R, Fiscella K, Xing G, et al. Determinants of patientoncologist prognostic discordance in advanced cancer. JAMA Oncol 2016;2(11):1421-1426

13 Epstein AS, Prigerson HG, O'Reilly EM, Maciejewski PK. Discussions of life expectancy and changes in illness understanding in patients with advanced cancer. J Clin Oncol 2016;34(20):2398-2403

14 Tanco K, Rhondali W, Perez-Cruz P, et al. Patient perception of physician compassion after a more optimistic vs a less optimistic message: a randomized clinical trial.JAMA Oncol 2015;1(02):176-183

15 van Vliet LM, Epstein AS. Current state of the art and science of patient-clinician communication in progressive disease: patients' need to know and need to feel known. J Clin Oncol 2014;32(31): 3474-3478

16 Temel JS, Greer JA, Admane S, et al. Longitudinal perceptions of prognosis and goals of therapy in patients with metastatic nonsmall-cell lung cancer: results of a randomized study of early palliative care. J Clin Oncol 2011;29(17):2319-2326

17 Morrison RS, Penrod JD, Cassel JB, et al; Palliative Care Leadership Centers' Outcomes Group. Cost savings associated with US hospital palliative care consultation programs. Arch Intern Med 2008;168(16):1783-1790

18 Zhang B, Wright AA, Huskamp HA, et al. Health care costs in the last week of life: associations with end-of-life conversations. Arch Intern Med 2009;169(05):480-488

19 Saito AM, Landrum MB, Neville BA, Ayanian JZ, Earle CC. The effect on survival of continuing chemotherapy to near death. BMC Palliat Care 2011;10:14
20 Leong M, Shah M, Smith TJ. How to avoid late chemotherapy. J Oncol Pract 2016;12(12):1208-1210

21 Harrington SE, Smith TJ. The role of chemotherapy at the end of life: “when is enough, enough?" JAMA 2008;299(22):2667-2678

22 Brumley R, Enguidanos S, Jamison P, et al. Increased satisfaction with care and lower costs: results of a randomized trial of inhome palliative care. J Am Geriatr Soc 2007;55(07):993-1000

23 Bakitas M, Lyons KD, Hegel MT, et al. Effects of a palliative care intervention on clinical outcomes in patients with advanced cancer: the Project ENABLE II randomized controlled trial. JAMA 2009;302(07):741-749

24 Zimmermann C, Swami N, Krzyzanowska M, et al. Early palliative care for patients with advanced cancer: a cluster-randomised controlled trial. Lancet 2014;383(9930):1721-1730

25 Temel JS, Greer JA, Muzikansky A, et al. Early palliative care for patients with metastatic non-small-cell lung cancer. N Engl J Med 2010;363(08):733-742

26 Temel JS, Greer JA, El-Jawahri A, et al. Effects of early integrated palliative care in patients with lung and GI cancer: a randomized clinical trial. J Clin Oncol 2017;35(08):834-841

27 Ferrell BR, Temel JS, Temin S, et al. Integration of palliative care into standard oncology care: American Society of Clinical Oncology Clinical Practice Guideline Update.J Clin Oncol 2017;35(01):96-112

28 Quill TE, Abernethy AP. Generalist plus specialist palliative carecreating a more sustainable model. N Engl J Med 2013;368(13): 1173-1175

29 Kamal AH, Gradison M, Maguire JM, Taylor D, Abernethy AP. Quality measures for palliative care in patients with cancer: a systematic review. J Oncol Pract 2014;10(04):281-287

30 Bickel KE, McNiff K, Buss MK, et al. Defining high-quality palliative care in oncology practice: an American Society of Clinical Oncology/American Academy of Hospice and Palliative Medicine Guidance Statement. J Oncol Pract 2016;12(09):e828-e838

31 Hui D, Finlay E, Buss MK, Prommer EE, Bruera E. Palliative oncologists: specialists in the science and art of patient care. J Clin Oncol 2015;33(20):2314-2317

32 Committee on Approaching Death. Addressing Key End of Life Issues, Institute of Medicine. Dying in America: Improving Quality and Honoring Individual Preferences Near the End of Life. Washington, DC: National Academies Press (US); 2015

33 Hui D, Elsayem A, De la Cruz M, et al. Availability and integration of palliative care at US cancer centers. JAMA 2010;303(11):1054-1061

34 Calton BA, Alvarez-Perez A, Portman DG, Ramchandran KJ, Sugalski J, Rabow MW. The current state of palliative care for patients cared for at leading US Cancer Centers: The 2015 NCCN Palliative Care Survey. J Natl Compr Canc Netw 2016;14 (07):859-866

35 Hui D, Bansal S, Strasser F, et al. Indicators of integration of oncology and palliative care programs: an international consensus. Ann Oncol 2015;26(09):1953-1959

36 Spetz J, Dudley N, Trupin L, Rogers M, Meier DE, Dumanovsky T. Few hospital palliative care programs meet national staffing recommendations. Health Aff (Millwood) 2016;35(09):1690-1697

37 Temel JS, Shaw AT, Greer JA. Challenge of prognostic uncertainty in the modern era of cancer therapeutics. J Clin Oncol 2016: JCO678573

38 Roeland EJ. Tailoring palliative care to the changing needs of people facing cancer. J Clin Oncol 2017;35(08):813-815

39 Freireich EJ, Kurzrock R. The role of investigational therapy in management of patients with advanced metastatic malignancy. J Clin Oncol 2009;27(02):304-306

40 Schapira L, Moynihan TJ, von Gunten CF, Smith TJ. Phase I versus palliative care: striking the right balance. J Clin Oncol 2009;27 (02):307-308

41 Hui D, Parsons H, Nguyen L, et al. Timing of palliative care referral and symptom burden in phase 1 cancer patients: a retrospective cohort study. Cancer 2010;116(18):4402-4409 
42 Cheng JD, Hitt J, Koczwara B, et al. Impact of quality of life on patient expectations regarding phase I clinical trials. J Clin Oncol 2000;18(02):421-428

43 Finlay E, Lu HL, Henderson HR, O’Dwyer PJ, Casarett DJ. Do phase 1 patients have greater needs for palliative care compared with other cancer patients? Cancer 2009;115(02):446-453

44 Sun V, Cooke L, Chung V, Uman G, Smith TJ, Ferrell B. Feasibility of a palliative care intervention for cancer patients in Phase I clinical trials. J Palliat Med 2014;17(12):1365-1368

45 Levine DR, Johnson LM, Mandrell BN, et al. Does phase 1 trial enrollment preclude quality end-of-life care? Phase 1 trial enrollment and end-of-life care characteristics in children with cancer. Cancer 2015;121(09):1508-1512

46 Ferrell BR, Chung VM, Koczywas M, et al. Integration of palliative care for patients with solid tumors on phase I clinical trials. J Clin Oncol 2016;34:138

47 Coldwell DM, Sewell PE. The expanding role of interventional radiology in the supportive care of the oncology patient: from diagnosis to therapy. Semin Oncol 2005;32(02):169-173

48 Tam A, Ahrar K. Palliative interventions for pain in cancer patients. Semin Intervent Radiol 2007;24(04):419-429

49 Chu L, Hawley P, Munk P, Mallinson P, Clarkson P. Minimally invasive palliative procedures in oncology: a review of a multi- disciplinary collaboration. Support Care Cancer 2015;23(06): 1589-1596

50 Fairchild AH, Rilling WS. Palliative interventional oncology. Cancer J 2016;22(06):411-417

51 Adam A, Kenny LM. Interventional oncology in multidisciplinary cancer treatment in the 21(st) century. Nat Rev Clin Oncol 2015; 12(02):105-113

52 Campbell TC, Roenn JH. Palliative care for interventional radiology: an oncologist's perspective. Semin Intervent Radiol 2007;24 (04):375-381

53 Daly ME, Riess JW. Multidisciplinary care. Cancer Treat Res 2016; 170:285-300

54 Monsky WL, Khorsand D, Nolan T, Douglas D, Khanna P. Quality of life assessment in interventional radiology. Acad Radiol 2014;21 (03):407-414

55 McCullough HK, Bain RM, Clark HP, Requarth JA. The radiologist as a palliative care subspecialist: providing symptom relief when cure is not possible. AJR Am J Roentgenol 2011;196(02):462-467

56 Requarth JA. IR and palliative care: a good match. J Vasc Interv Radiol 2015;26(11):1740-1741

57 Dharmarajan KV, Wei R, Vapiwala N. Primary palliative care education in specialty oncology training: more work is needed. JAMA Oncol 2016;2(07):858-859 